\title{
Transatlantica
}

Revue d'études américaines. American Studies Journal

2 | 2017

(Hi)stories of American Women: Writings and Rewritings / Call and Answer: Dialoguing the American

West in France

\section{Hommage à François Brunet}

\section{Jean Kempf}

\section{OpenEdition}

\section{Journals}

Édition électronique

URL : https://journals.openedition.org/transatlantica/11293

DOI : 10.4000/transatlantica. 11293

ISSN : 1765-2766

Éditeur

Association française d'Etudes Américaines (AFEA)

Référence électronique

Jean Kempf, « Hommage à François Brunet », Transatlantica [En ligne], 2 | 2017, mis en ligne le 22 mai 2019, consulté le 02 février 2023. URL : http://journals.openedition.org/transatlantica/11293; DOI : https://doi.org/10.4000/transatlantica.11293

Ce document a été généré automatiquement le 2 février 2023

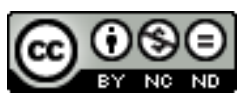

Creative Commons - Attribution - Pas d'Utilisation Commerciale - Pas de Modification 4.0 International - CC BY-NC-ND 4.0

https://creativecommons.org/licenses/by-nc-nd/4.0/ 


\title{
Hommage à François Brunet
}

\author{
Jean Kempf
}

1 François Brunet était mon ami, mais je souhaiterais dans ces quelques lignes laisser de côté la dimension personnelle pour évoquer le chercheur et l'intellectuel. Au-delà de ses relations individuelles, ce François Brunet-là appartient à tous, à tous ceux qui voudront le lire. Pour évoquer ici le chercheur, je reprendrai ici brièvement le fil de deux textes, l'introduction à L'Amérique des images (2013) et son dernier livre, La Photographie. Histoire et contre-histoire (2017) ${ }^{1}$. Je suis heureux qu'il me soit permis de le faire dans Transatlantica, une revue à laquelle François Brunet était très attaché. Lors de la naissance du projet dans les années 2000, nous avions longuement discuté, et je me souviens que, outre la possibilité de publier une iconographie riche et variée (ce qui restait un problème presque insurmontable pour nos revues papier), l'idée d'une publication en ligne le séduisait en raison des nouvelles formes de rendu qu'elle permettait d'envisager. Moins se préoccuper des formats canoniques (qu'il ne méprisait nullement et qu'il pratiquait avec bonheur), permettre d'aller de la publication-édition de textes inédits à un bref commentaire d'image, élargir les modalités d'intervention du savoir universitaire et pouvoir utiliser ces savoirs plus directement dans les cours, voilà qui allait bien dans le sens de sa conception du métier de chercheur enseignant. Même si les réalités du droit commercial devaient nous obliger à en rabattre sur les espoirs d'origine en matière de diffusion des images, et même si les contraintes de la pratique universitaire n'ont pas toujours rendu possible toutes les expérimentations dont nous rêvions alors, François Brunet est resté un collaborateur attentif et engagé de Transatlantica, participant au comité de rédaction, conseillant les uns et les autres, aidant à la publication de textes de jeunes auteurs et encourageant le développement de rubriques nouvelles comme Trans'Art.

2 Toute l'œuvre de François Brunet tourne autour de l'idée que penser la culture, c'est penser l'image, ce qui, aujourd'hui plus que jamais, ne saurait se faire sans penser à la fois l'histoire des images et les images comme histoire. Ce qui pourrait passer, dans ce raccourci, comme une énième reformulation de la posture classique des cultural studies (américaines tout au moins) est en fait un déplacement important que développe Histoire et contre-histoire. Les cultural studies, que François Brunet connaissait bien, gardaient du marxisme une forme de mécanisme originel qui ne satisfaisait pas 
totalement son désir de comprendre comment l'on passe de la séduction des images à leur caractère structurant d'une société. Dix-neuviémiste spécialiste des États-Unis, François Brunet constatait qu'un monde protestant, donc plutôt iconoclaste, était devenu la société la plus iconophile qui soit sans pour autant jamais se départir de la fascination de la lettre (nous ne cessions de dire qu'il faudrait écrire quelque chose sur le fait que, par exemple, les panneaux de signalisation américains préfèrent bien souvent le texte au pictogramme). Cette question, qui est à la base des visual studies (Boidy), François Brunet n'a cessé de la poser, et c'est elle qui a guidé l'évolution de son travail.

3 Historien de la naissance de la photographie dans ses premiers travaux, il a peu à peu embrassé la totalité de l'histoire de la photographie qu'il a éclairée, jusqu'au présent le plus contemporain, de sa connaissance non seulement des origines de l'idée de photographie, titre de son livre récemment republié (2012), mais surtout des interrogations anciennes sur l'image. Comme un François Hartog a pu penser le présentisme à partir de sa formation d'antiquisant, François Brunet s'est nourri de sa formation de philologue et de classiciste pour comprendre ce que la photographie faisait à l'image. Dans le même temps, il restait, par goût certainement, mais aussi par conviction intellectuelle, attaché à une histoire des sciences et à des considérations " concrètes » : la circulation des images et, d'une brûlante actualité, « le contrôle quasi orwellien qu'exercent certains grands groupes sur le "droit" de citation" (2013 12). Aussi ne se plaçait-il ni dans une ontologie de la photographie, qui aurait en quelque sorte épuisé le sens du médium, ni dans une manière de réductionnisme, qui aurait fait de toutes les images un seul et même objet conceptuel. Si les posters, les timbres-poste et les cartes postales sont bien des images, il convient toutefois, disait-il, de les distinguer, comme il le montre dans sa subtile discussion du poster d'Obama (« Hope ») dans l'introduction de L'Amérique des images (2013 9-11). C'est ce décalage qui lui faisait à la fois apprécier et défendre les visual studies et l'idée d'un pictorial turn, mais aussi s'en démarquer sensiblement. Excellent connaisseur de la littérature sur la question, tout particulièrement de l'un de ses hérauts, W.J.T. Mitchell, il n'en épousait pas pour autant le programme, en tout cas pas totalement, comme on peut le voir dans l'article qu'il avait consacré à une présentation de cette "école » américaine (2005). Dans ce texte, et ailleurs, on perçoit bien la distance qu'il prenait à la fois par rapport à la nature systématique du propos (la systématique le mettait toujours sur ses gardes) et au côté par trop «situé » de ces études - le contexte réflexif de chercheurs étatsuniens travaillant sur leur propre pays (2005 91-92). Selon lui, en effet, ces chercheurs ne se départissent pas «toujours d'un penchant essentialiste qui prédit une destinée iconique "naturelle" à la culture nationale » (2013 7). Ce dernier point est loin d'être négligeable car il illustre parfaitement ce que des américanistes non étatsuniens peuvent et doivent apporter au débat : les études de François Brunet sur la naissance de l'idée de photographie doivent beaucoup à la philosophie européenne et à un dialogue franco-britannique - probablement plus qu'à Emerson et James.

Quand on regarde dans le détail le travail de François Brunet, on est en effet frappé de constater combien celui-ci, au-delà d'une recherche sur les images, est aussi un exercice de mise en dialogue de traditions épistémologiques différentes, lorsqu'il est capable de faire tenir dans le même texte Peirce et Barthes, ou Sontag et Rancière, sans que pour autant l'un ne soit réduit à l'autre dans un syncrétisme brouillon. François Brunet aimait relire des textes oubliés qu'il prenait au sérieux : par exemple le livre de Daniel Boorstin, The Image: A Guide to Pseudo-events in America (1961) ou celui de Robert 
Taft, Photography and the American Scene (1938). Il débute d'ailleurs Histoire et contrehistoire par un texte qui le fascinait, le discours de Paul Valéry pour le centenaire de la photographie (2017 11-12). Ceci le conduit à formuler l'hypothèse centrale, et à mon sens capitale, selon laquelle émergent dans les années 1930 deux conceptions de la photographie: l'une internaliste, celle de la construction du médium, l'autre externaliste, celle de "l'investigation [des] archives en tant qu'elles fournissent des images ou des imaginaires du passé » (2017 15-16). C'est déjà cette idée qu'il exprimait dans l'introduction à L'Amérique des images, en y ajoutant une dimension de circulation transatlantique : «l'affinité entre Amérique et image n'est ni naturelle ni banale ; [...] elle est le produit d'une histoire et d'une culture». Il s'agit donc "de répondre à la métaphysique de l'Amérique-image par l'histoire des formes et des pratiques de l'image aux États-Unis [...]. Et à la critique platonicienne des simulacres par l'analyse des images en tant que culture » (2013 7-8).

5 Pour penser cette métahistoire des images, François Brunet s'était fait historiographe dans une tradition qui n'écarte pas les textes perçus comme faux ou dépassés (version intellectuelle de la tabula rasa américaine), mais élabore sur eux des commentaires infinis. Ce qui l'a conduit à proposer une formulation dialectique de l'histoire des images, celle d'une tension fondatrice entre deux histoires comprises à la fois comme deux conceptions de la nature de l'image photographique et deux narrations de ce que la photographie apporte ou «fait»- pour reprendre la question de Mitchell : "Que veulent les images?». Il entend ainsi éclairer notre présent politique où est apparue, après l'époque de l'opposition vérité/mensonge, celle de la "post-vérité » et des énoncés publics relevant d'espaces parallèles dans lesquels se développent des « vérités concurrentes ». C'est à mon sens ce qu'il voyait dans les débats qui agitent le milieu de la photographie autour de la "véracité des photos ", débat qu'il aborde de front dans son Histoire, contre histoire à travers un autre livre, celui d'Errol Morris (2011), mais aussi à travers la controverse sur les images du débarquement de Robert Capa (Brunet, 2017 318-319). Il ne faut donc pas se méprendre sur le titre : "histoire et contre-histoire", comme s'il existait - c'est trop souvent le cas dans nos disciplines - un discours hégémonique, d'un côté, que des révisionnistes en chevaliers blancs de la vérité viendraient subvertir, de l'autre. Ici, histoire et contre-histoire sont indissociables, voire imbriquées - elles s'interpénètrent, dit-il (15) - et ne pas les tenir ensemble nous empêchera de comprendre notre présent.

Ces quelques coups de projecteur sur une démarche qui est à mes yeux originale (parce que profondément informée de ce qui l'a précédé) et féconde (parce qu'elle nous pousse vers des champs nouveaux) n'épuisent pas, loin s'en faut, tous les aspects du travail de François Brunet, mais j'espère qu'ils donneront l'envie de lire ou relire ses textes, des textes où l'on découvrira son style limpide et sans afféterie lexicale, où la période est courte sans être sèche, où l'inférence est implacable sans être jamais visible autrement que par la voix qui entraîne le lecteur dans la démonstration (« rien qui pèse ou qui pose »).

7 Mais je ne voudrais pas conclure ces lignes sans évoquer un mot capital pour lui : les archives. Il s'agit bien d'un pluriel, car l'Archive est un terme qu'il trouvait trop conceptuel et dont, par conséquent, il se méfiait. François Brunet était en effet un historien au sens large et plein du terme - il faut ici rappeler l'influence qu'ont eu très tôt sur lui son oncle, Jacques Lefort, enseignant à l'École Pratique des Hautes Études, éditeur des manuscrits du Mont Athos, et sa passion pour les archives grandes ou 
petites (l'un de ses derniers travaux a été l'édition des archives de son arrière-grandpère). Dès que ses voyages aux États-Unis lui en laissaient le temps, il s'y plongeait quelques jours ou quelques heures, et l'un de ses regrets était de ne pas pouvoir y consacrer assez de temps. Ce temps qui lui a manqué, il était en train de le rattraper, lorsque la mort l'a saisi, avec ce projet franco-américain autour de l'exposition universelle de 1867. Projet ambitieux qui voulait montrer la bascule que cet événement avait produite dans la perception que le public européen avait de l'Amérique, lorsqu'au contact des images concrètes et des artefacts venant des États-Unis, le terme autrefois péjoratif $\mathrm{d}^{\prime}$ " images américaines " (c'est-à-dire produit commercial et donc indigne face à la vraie culture) s'effaça devant la matérialité presque sidérante des images réelles. Cet ambitieux projet qui, je l'espère, sera porté à son terme par celles et ceux qui travaillaient avec lui, caractérisait bien un autre aspect de sa démarche : d'un côté, comprendre le rapport entre les spectateurs et les images et, de l'autre, communiquer ce savoir au public d'aujourd'hui. La passion encyclopédique qui animait François Brunet, avec ce que cette passion peut avoir de véritablement charnel et de dévorant, est une dimension importante de son rapport au rôle et au travail du chercheur qui est aussi un intellectuel. Si le temps lui a manqué pour se plonger encore davantage dans les archives, c'est enfin, il me semble indispensable de le rappeler, parce qu'il consacrait une large partie de son énergie à la vie universitaire, en assumant de lourdes charges collectives, en tant que directeur du laboratoire de recherche sur les cultures anglophones (LARCA) à l'Université Paris-Diderot notamment, et à la formation. Les jeunes chercheurs et chercheuses qu'il a conduit•e•s dans leurs premiers travaux et pour certain•e•s jusqu'à la thèse, et dont il me répétait souvent combien il les trouvait stimulant•e•s, inventif•ve•s, et «formidables » - lui qui était déjà tout cela - arrivent aujourd'hui au seuil de la carrière (certain•e•s y sont déjà entré•e•s) et, j'en suis persuadé, porteront ses exigences et sa vision dans leur propre travail.

\section{BIBLIOGRAPHIE}

La bibliographie de François Brunet se trouve en partie sur le site de HAL-SHS : https:// cv.archives-ouvertes.fr/brunet-francois

BOIDY, Maxime. Les études visuelles. Saint-Denis : Presses de l'Université de Vincennes-SaintDenis, 2017.

BOORSTIN, Daniel. The Image: A Guide to Pseudo-events in America. New York : Harper \& Row, 1961.

BRUNET, François. « Théorie et politique des images. W.J.T. Mitchell et les études de visual culture ». Études anglaises, n 58, 2005, p. 82-93.

---. La naissance de l'idée de photographie. 2000. Paris : Presses Universitaires de France, 2012.

---, dir. L'Amérique des images. Histoire et culture visuelles des États-Unis. Paris : Hazan, 2013.

---. La photographie. Histoire et contre-histoire. Paris : Presses Universitaires de France, 2017. 
MORRIS, Errol. Believing is Seeing: Observations on the Mysteries of Photography. New York : Penguin, 2011.

TAFT, Robert. Photography and the American Scene: A Social History, 1839-1889. New York : Macmillan, 1938.

\section{NOTES}

1. Qu'il me soit ici permis cependant une remarque personnelle qui éclaire le lien entre les deux textes. François Brunet écrivait dans sa dédicace à La Photographie. Histoire et contre-histoire, qu'il m'avait envoyé à sa parution, « Encore mon Amérique des images en fait. »

\section{AUTEUR}

\section{JEAN KEMPF}

Université Lumière-Lyon 2 